\title{
Inducción de membrana y trasposición de peroné ipsolateral no vascularizado en neoformación ósea postraumática. Reporte de caso
}

\author{
Bustos-Mora R, * Betancourt CM,** Valadez-Soto JM, ${ }^{* *}$ Flores-Villalobos A** \\ IMSS Hospital de Especialidades del Centro Médico de Occidente
}

RESUMEN. Objetivo: Demostrar los resultados en la neoformación ósea de tibia utilizando la técnica de inducción de membrana más la transposición de peroné ipsolateral no vascularizado. Caso clínico: Masculino de 25 años, antecedente de sufrir impacto por arma de fuego en pierna izquierda dos años antes, presentó un diagnóstico inicial de fractura expuesta Gustilo IIIB AO 42 C3 IO4NV1MT2 con pérdida ósea de $7 \mathrm{~cm}$ de diáfisis tibial. Durante su evolución se realizaron múltiples aseos quirúrgicos y colocación de injertos cutáneos con resultados no favorables, por lo que se emplea la técnica de membrana inducida más colocación de peroné ipsolateral no vascularizado, teniendo una evolución a los cuatro meses posterior a su última intervención con deambulación dependiente de bastón. Conclusión: La técnica de inducción de membrana con transposición de peroné ipsolateral no vascularizado podría ser una alternativa adecuada para el manejo de pérdidas óseas en los pacientes.

Palabras clave: Membrana inducida, peroné no vascularizado, pérdida ósea.

\footnotetext{
* Médico Especialista en Traumatología y Ortopedia. Profesor titular de Postgrado en Traumatología y Ortopedia, Médico adscrito al módulo de politrauma.

** Residente de la Especialidad de Traumatología y Ortopedia.
}

Hospital de Especialidades del Centro Médico de Occidente del IMSS en Guadalajara, Jalisco.

Dirección para correspondencia:

Rafael Bustos Mora

Belisario Domínguez Núm. 1000,

Col. Independencia Oriente, CP 44340,

Guadalajara, Jalisco, México.

E-mail: dr.rafaelbustos@gmail.com

Este artículo puede ser consultado en versión completa en http:// www.medigraphic.com/actaortopedica
ABSTRACT. Objective: To demonstrate the outcome of tibial bone neo formation, using induced-membrane technique and nonvascularized ipsolateral fibular graft transposition. Case report: A 25 years old male with a 2 years ago firearm injury in left leg, presenting an initial diagnosis of open fracture Gustilo IIIB AO $42 \mathrm{C} 3$ IO4NV1MT2 with a $7 \mathrm{~cm}$ tibial diaphyseal bone defect. During his hospital evolution multiple interventions were made including surgical debridement and skin grafts placement, with unfavorable results. Therefore, we decided to use the induced-membrane technique and nonvascularized ipsolateral fibular graft transposition, resulting in a cane dependent ambulation, in 4 months evolution after last intervention. Conclusion: Induced-membrane technique and non-vascularized ipsolateral fibular graft transposition could be a successful alternative for the management of patients with severe bone loss.

Key words: Induced-membrane, non-vascular fibular graft, bone defect.

\section{Introducción}

La reconstrucción de grandes defectos de huesos largos a menudo es el principal desafío en el salvamento de las extremidades, sin importar la etiología de la pérdida ósea. ${ }^{1}$

A través del tiempo se han empleado distintas técnicas para que se realice la neoformación ósea adecuada. En 1986 Masquelet describió un procedimiento de dos etapas en el que una membrana de tejido inducida por un espaciador de cemento en un defecto óseo facilitó la consolidación del injerto óseo y por lo tanto, evitó la reabsorción. ${ }^{2}$ La primera etapa implica el desbridamiento de todo el tejido desvitalizado, utilizando reducción abierta y fijación interna y la colocación de un espaciador de cemento con o sin antibióticos. En la segunda etapa, que se realiza al menos seis semanas después de la anterior, se retira el espaciador y el vacío resultante se llena con injerto óseo. ${ }^{3}$ 
Las principales propiedades de la membrana son prevenir la reabsorción del injerto y secretar factores de crecimiento. La membrana inducida aparece como una cámara biológica, lo que permite la concepción de numerosos modelos experimentales de reconstrucción ósea. Este concepto probablemente podría extenderse a otras reparaciones de tejidos. ${ }^{1}$

Por otra parte, los cirujanos ortopédicos tienen la opción de elegir transferencias óseas vascularizadas, transporte óseo, injertos óseos alogénicos, sustitutos del injerto óseo y muchos otros medios para tratar las pérdidas de tejido óseo. El injerto de peroné no vascularizado y el injerto de hueso esponjoso proporcionan un medio confiable para tratar tales afecciones en los países en vías de desarrollo. ${ }^{4}$ Dentro de estas opciones, el estándar de oro en la literatura es el hueso esponjoso cosechado de la crestas ilíacas. ${ }^{5}$

El injerto peroneo es un método frecuentemente manipulado en los defectos creados por escisiones tumorales, pérdidas traumáticas o por secuelas de infección en pediatría, ${ }^{6}$ su uso se origina a principios del siglo XX y fue el estándar de oro para la reconstrucción biológica por más de 60 años, principalmente para lesiones de origen neoplásico o infeccioso. ${ }^{7}$

El peroné es utilizado debido a su fácil acceso y mínima morbilidad para el sitio donador, ${ }^{6}$ sobre todo por: su excelente y resistente estructura ósea, potencial osteogénico importante, se obtiene mediante procedimiento más sencillo, evita el uso de costosos implantes y no afecta de manera considerable el sitio donante. A diferencia de los aloinjertos no tiene problemas de inmunogenicidad ni riesgos de transmisión de infección, ${ }^{8}$ por lo que se ha vuelto una práctica solución para los defectos óseos en hospitales localizados en los países en vías de desarrollo. ${ }^{9}$

Se ha observado que los defectos mayores de 5 a $7 \mathrm{~cm}$, por lo general no deben ser candidatos a un injerto peroneo no vascularizado. Sin embargo, el origen de este parámetro se respalda en evidencia no específica. ${ }^{10}$

El objetivo del presente estudio fue demostrar los resultados en la neoformación ósea tibial, utilizando la técnica de inducción de membrana más la transposición de peroné ispsolateral no vascularizado.

\section{Caso clínico}

Masculino de 25 años con antecedente de lesión por arma de fuego tipo retrocarga en cara anterolateral y tercio medio de pierna izquierda, con diagnóstico inicial de fractura de tibia izquierda expuesta con pérdida ósea de $7 \mathrm{~cm}$, clasificación Gustilo IIIB, AO 42C3 IO4NV1MT2 (Figura 1).

Manejo inicial: se otorgó manejo inicial en un hospital auxiliar mediante control de daños con fijación externa y múltiples aseos quirúrgicos. Posterior a 20 días de hospitalización se envía al hospital de especialidades para establecer tratamiento definitivo.

Manejo en el hospital de especialidades: se observó a su ingreso pérdida de tejido músculo tegumentario en el compartimento anterolateral del tercio proximal y medio diafisario tibial de aproximadamente $15 \mathrm{~cm}$ de diámetro, con exposición de fragmentos óseos (proximal y distal) y pérdida de hueso de aproximadamente 7-9 centímetros de diáfisis tibial, con abundante tejido necrótico circundante y tejido de granulación en tejido celular subcutáneo debido a las terapias de presión negativa proporcionadas previamente (Figura 2). Durante los cuatro primeros meses de hospitalización se realizaron múltiples aseos quirúrgicos y la colocación de injerto cutáneo.

Primera intervención quirúrgica. Al término de los primeros cuatro meses se realizó el primer intento de la técnica de inducción de membrana (colocación de injerto cutáneo y posteriormente la colocación del espaciador de cemento),
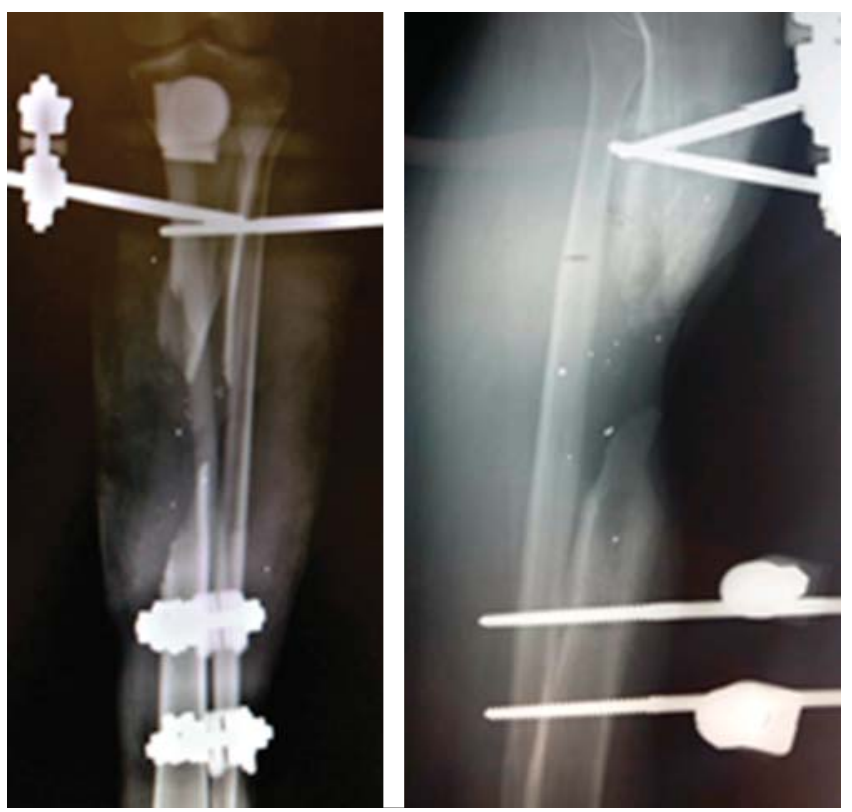

Figura 1: Radiografía AP y lateral que muestra la fractura de tibia con pérdida ósea de $7 \mathrm{~cm}$.

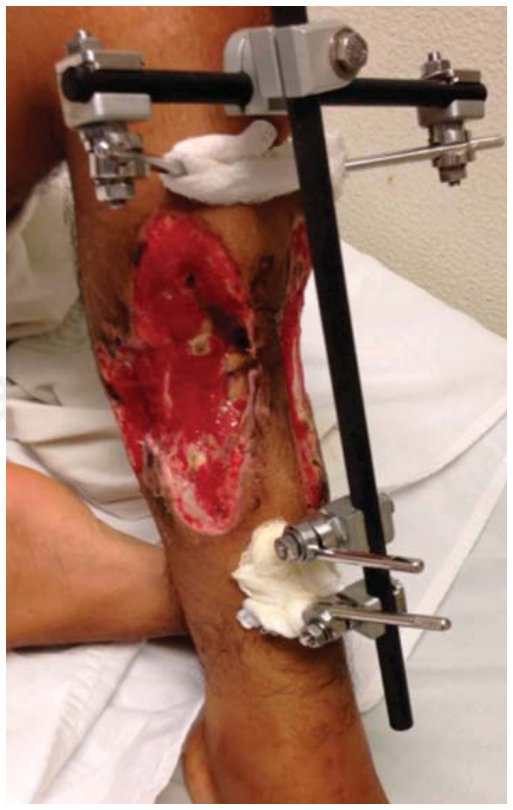

Figura 2:

Lesión músculo-tegumentaria posterior a realizar múltiples aseos en hospital de primera atención. 
teniendo una evolución tórpida caracterizada por necrosis tegumentaria del injerto y exposición del polimetilmetacrilato (PMMT) (Figura 3).

Primera reintervención: al haber presentado falla en la intervención inicial se realizó nuevamente la primera fase para la formación de membrana con rotación de colgajo de gastrocnemio medial e injerto cutáneo de muslo ipsolateral y la colocación de cemento óseo, evolucionando satisfactoriamente (Figura 4). Ocho semanas después se realizó la segunda fase colocando injerto óseo de cresta ilíaca, trasposición de $10 \mathrm{~cm}$ de peroné no vascularizado y matriz ósea desmineralizada con cierre de membrana (Figura 5); se le agregó una protección con fijación externa monoplanar.

Se retiró la fijación externa a los 10 meses. Al momento de deambular, el paciente presenta fractura lineal de la consolidación ósea proximal (Figura 6).

Segunda reintervención: la presencia de la fractura de consolidación motivó la realización de curetaje y reubicación del injerto de peroné proximal con protección de placa LCP tibia proximal (Figura 7).

Cuatro meses después de la segunda reintervención, el paciente mostró consolidación completa, con marcha dependiente de bastón y el miembro pélvico izquierdo con heridas afrontadas, sin datos de infección.

\section{Técnica}

Primera fase de inducción de membrana posterior a complicación por necrosis tegumentaria y exposición de PMMT: decúbito supino, anestesia regional, asepsia antisepsia, debridación de tejido necrótico, retiro de PMMT y aseo abundante. Se observó pérdida ósea de aproximadamente $7 \mathrm{~cm}$ en el tercio proximal con medio diafisario de tibia, con pérdida de músculo tegumentaria circunferencial de $13 \mathrm{~cm}$, se realizó preparación con canalización de

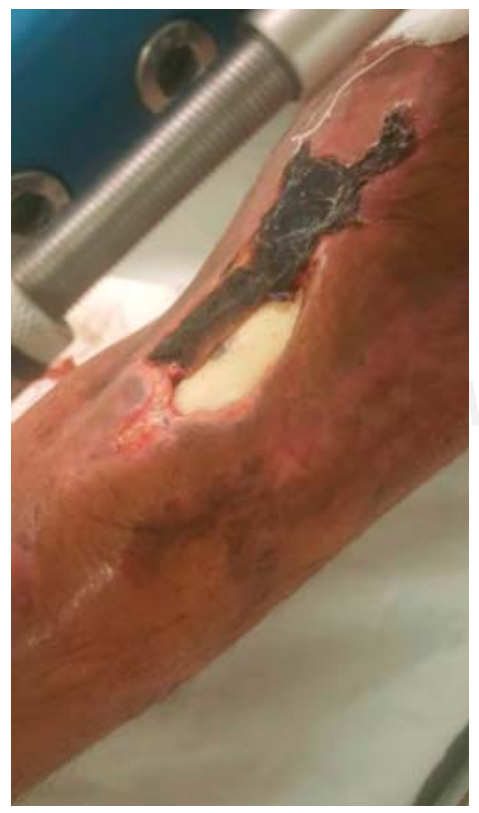

Figura 3:

Complicación de necrosis de injerto cutáneo con exposición de polimetilmetacrilato. fragmentos óseos, se colocó fijación externa monoplanar con preservación de espacio óseo de $7 \mathrm{~cm}$, se preservó el espacio con PMMT en forma cilíndrica de $7 \mathrm{~cm}$, se hizo incisión de $20 \mathrm{~cm}$ en cara medial de pierna para la rotación de porción medial de gastrocnemio medial, se realizó cobertura del colgajo con injerto de piel de muslo izquierdo de aproximadamente $12 \mathrm{~cm}$ por $10 \mathrm{~cm}$.

Segunda fase de inducción de membrana más colocación de peroné no vascularizado: mediante abordaje lateral tercio medio diafisario de peroné se extrajeron $10 \mathrm{~cm}$ de peroné no vascularizado respetando periostio. Posteriormente se
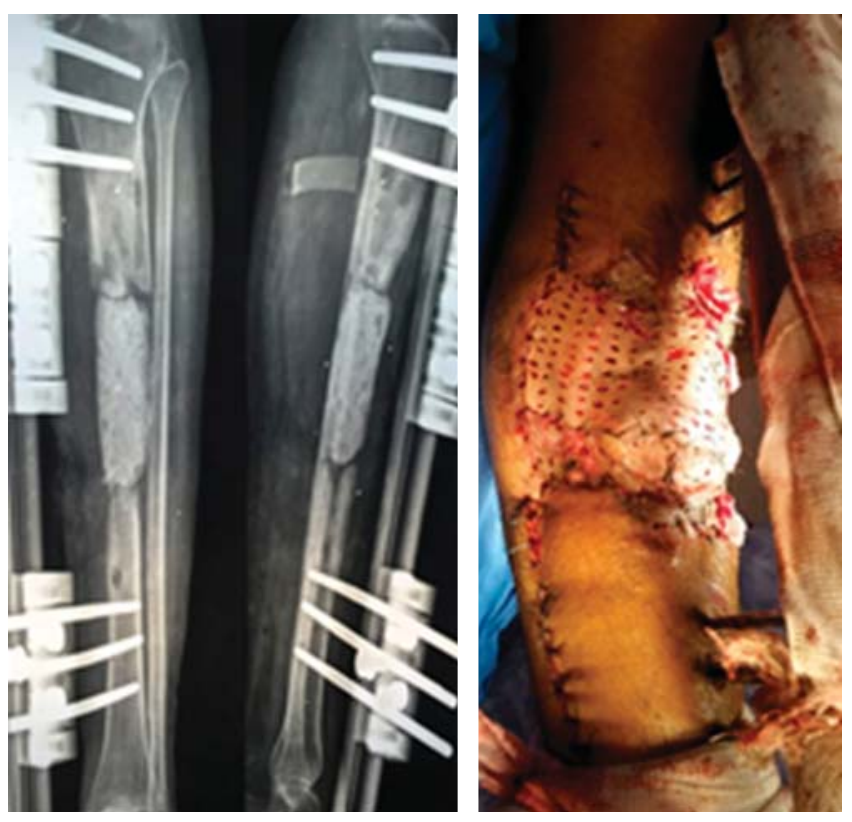

Figura 4: Resultado de nueva colocación de primer tiempo de colocación de PMMC con rotación de colgajo de gastronemio medial y cobertura de injerto cutáneo.

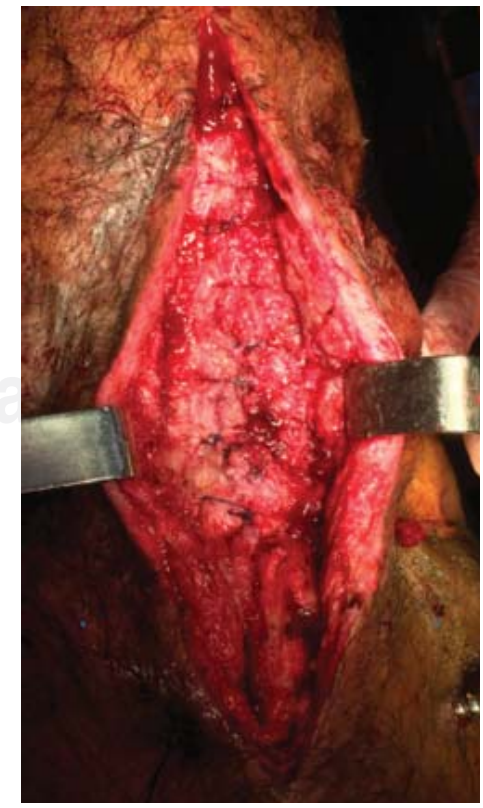

Figura 5:

Segundo tiempo con colocación de peroné e injerto de cresta, mostrando cierre de tejido circundante a membrana. 


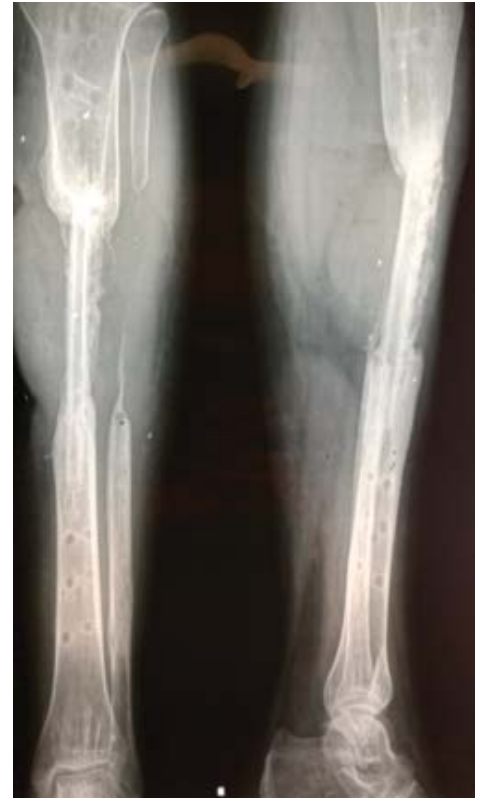

Figura 6:

Muestra sitio de fractura en consolidación peroneotibial proximal.

realizó abordaje medial diafisario tibial respetando colgajo musculocutáneo de $15 \mathrm{~cm}$, se extrajo el PMMT, se recanalizaron los fragmentos óseos proximal y distal, se colocó transposición de peroné no vascularizado más injerto de cresta y matriz ósea $10 \mathrm{~cm}^{3}$, a continuación cierre cuidadoso de membrana inducida con monocryl.

\section{Discusión}

Las pérdidas óseas postraumáticas son un reto para el cirujano ortopedista, en el presente estudio se valoró el uso combinado de la técnica de inducción de membrana con la inclusión de la trasposición de peroné ipsolateral no vascularizado con un defecto postraumático de $7 \mathrm{~cm}$.

Se utilizó el autoinjerto de peroné no vascularizado (libre) debido a que ha demostrado ser un método tan útil y confiable como el injerto vascularizado en lesiones de resección ósea por presencia de neoplasias. ${ }^{11}$

En la actualidad se han discutido principalmente dos importantes limitaciones de la técnica tradicional de Masquelet: a) la cantidad y fuente de injerto esponjoso necesaria para rellenar adecuadamente el defecto óseo recubierto por la membrana inducida y b) el tiempo promedio para su consolidación que es de ocho meses aproximadamente, ${ }^{12}$ lo cual a menudo hace que su uso se vea limitado. Otras de las complicaciones más frecuentemente reportadas en la literatura para la técnica de inducción de membrana son: consolidación parcial, necesidad de un injerto secundario o/y mal alineamiento en las extremidades. ${ }^{2}$

La colocación de autoinjerto de peroné podría disminuir notablemente la necesidad de hueso esponjoso y otorgar un mejor soporte para el apoyo precoz como lo fue en el presente caso.

Una consideración importante a tomar en cuenta para el paciente es el tiempo de apoyo que depende de cada una de las técnicas: en el caso de la realizada por Masquelet se recomienda el apoyo parcial entre el cuarto y quinto mes y la

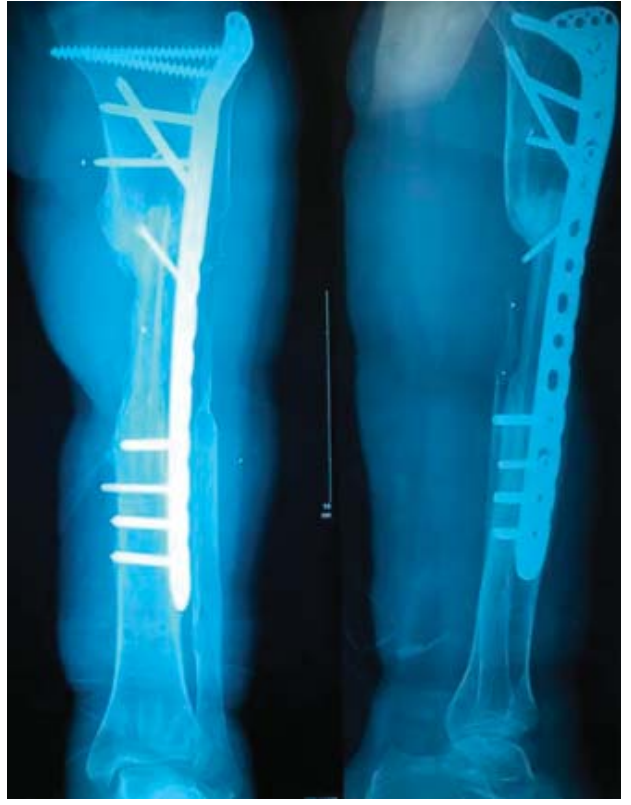

Figura 7: Resultado de reintervención de osteosíntesis de fractura de consolidación proximal con placa LCP tibia proximal.

carga completa entre el sexto y octavo mes. ${ }^{13}$ Por otra parte, en la trasposición de peroné no vascularizado se ha reportado apoyo a partir de la vigésima semana al retirar el fijador externo, alcanzando datos de consolidación radiológica completa a los 12 meses y una hipertrofia y/o tibialización del peroné a los 4.5 años. ${ }^{14}$

A los cuatro meses de la última intervención por fractura en sitio de anastomosis ósea el paciente en estudio mostró deambular con apoyo únicamente de bastón.

\section{Conclusión}

La técnica de neoformación ósea combinando la utilización de membrana inducida con la transposición de peroné ipsolateral no vascularizado podría ser un abordaje quirúrgico ortopédico de fácil realización para los pacientes con pérdida ósea mayor de $5 \mathrm{~cm}$.

Bibliografía

1. Masquelet AC, Begue T. The concept of induced membrane for reconstruction of long bone defects. Orthop Clin North Am. 2010; 41(1): 27-37.

2. Olesen UK, Eckardt H, Bosemark P, Paulsen AW, Dahl B, Hede A The Masquelet technique of induced membrane for healing of bone defects. A review of 8 cases. Injury. 2015; 46 Suppl 8: S44-7.

3. Konda SR, Gage M, Fisher N, Egol KA. Segmental bone defect treated with the induced membrane technique. J Orthop Trauma. 2017; 31(Suppl 3): S21-2.

4. Lawal YZ, Garba ES, Ogirima MO, Dahiru IL, Maitama MI, Abubakar K, et al. Use of non-vascularized autologous fibula strut graft in the treatment of segmental bone loss. Ann Afr Med. 2011; 10(1): 25-8.

5. Masquelet AC. Induced membrane technique: pearls and pitfalls. $J$ Orthop Trauma. 2017; 31(Suppl 5): S36-8. 
6. Swamy MK, Rathi A, Gupta V. Results of non-vascularised fibular grafting in gap non-union of long bones in paediatric age group. J Clin Orthop Trauma. 2013; 4(4): 180-4.

7. Lenze U, Kasal S, Hefti F, Krieg AH. Non-vascularised fibula grafts for reconstruction of segmental and hemicortical bone defects following meta/diaphyseal tumour resection at the extremities. BMC Musculoskelet Disord. 2017; 18(1): 289.

8. Morsi E. Tibial reconstruction using a non-vascularised fibular transfer. Int Orthop. 2002; 26(6): 377-80.

9. Thevarajan K, Teo P. Free non-vascularized fibular graft for treatment of pediatric traumatic radial bone loss: a case report. Malays Orthop J. 2013; 7(2): 37-40.

10. Allsopp BJ, Hunter-Smith DJ, Rozen WM. Vascularized versus nonvascularized bone grafts: what is the evidence? Clin Orthop Relat Res. 2016; 474(5): 1319-27.
11. Schuh R, Panotopoulos J, Puchner SE, Willegger, Hobusch GM, Windhager R, et al. Vascularised or non-vascularised autologous fibular grafting for the reconstruction of a diaphyseal bone defect after resection of a musculoskeletal tumour. Bone Joint J. 2014; 96-B(9): 1258-63.

12. Vejarano-Solano J, Ruiz-Semba C, Ganoza-Arróspide C, HurtadoFernández J. Reconstrucción de defectos óseos segmentarios postraumáticos mediante técnica de inducción de membrana. Rev Med Hered. 2015; 26(2): 76-85.

13. Autorino C. Membrana inducida (membrana de Masquelet) técnica para el tratamiento de defectos óseos segmentarios. ACARO (Internet). 2016; (1): 4-5.

14. El-Sayed M, El-Hadidi M, El-Adl W. Free non-vascularised fibular graft for treatment of post-traumatic bone defects. Acta Orthop Belg. 2007; 73(1): 70-6. 